\title{
Le rôle des urgentologues dans la désaccoutumance au tabac et le programme de lutte contre le tabagisme en Colombie-Britannique
}

\author{
Kai Wai Cheung, MD, MPH
}

Bien que les problèmes de santé publique tels que l'augmentation inquiétante de l'obésité aient fait l'objet d'une couverture médiatique, le tabagisme reste une cause importante de mortalité évitable au Canada. ${ }^{1}$ D'après l'Enquête de surveillance de l'usage du tabac au Canada menée en 2011, à peu près 5 millions de personnes âgées de 15 ans et plus au pays étaient fumeurs ${ }^{2}$; par ailleurs, une proportion encore plus grande de la population est exposée à des substances cancérogènes connues en raison de la fumée secondaire. Environ 37000 personnes meurent, chaque année, au Canada, par suite de l'exposition à la fumée de tabac. ${ }^{3}$ De plus, le tabagisme entraîne une forte morbidité, qui se traduit par des maladies chroniques, et elles-mêmes nécessitent des consultations fréquentes aux services des urgences (SU) et des hospitalisations. Le fardeau financier du tabagisme au Canada s'élève à 17 milliards de dollars par année, dont 4.4 milliards sont liés à des soins de santé directs. ${ }^{3}$

$\mathrm{Au}$ fil du temps, de nombreuses mesures ont été prises pour juguler la pandémie de maladies liées au tabagisme au Canada, notamment l'augmentation du coût des produits du tabac et des taxes sur ces biens, la mise en œuvre de politiques imposant l'organisation de lieux publics exempts de fumée, et des restrictions relatives à la publicité. En septembre 2011, le ministre de la Santé de la Colombie-Britannique (C.-B.) a mis sur pied un programme provincial de lutte contre le tabagisme. ${ }^{4}$ Le programme est accessible à tous les habitants de la province selon deux formules, soit la thérapie de remplacement de la nicotine (TRN), gratuite et sans ordonnance pour les fumeurs; soit des médicaments de désaccoutumance au tabac sur ordonnance, subventionnés en partie pour les fumeurs admissibles. Les deux médicaments assurés sont le bupropion (Zyban) et la varénicline (Champix). Les personnes peuvent se prévaloir du programme en composant le 811, et on leur offre, selon le cas, soit la TRN, soit un médicament sur ordonnance pour une durée de 12 semaines par année civile, délivré chaque fois en tranches de 28 jours. Certes, une étude récente a remis en question l'efficacité de la TRN, ${ }^{5}$ mais des méta-analyses ont démontré que la TRN doublait le taux d'abandon du tabac au bout de 6 mois $^{6,7}$ et que la varénicline $^{8}$ et le bupropion 9 augmentaient tous les deux d'un peu plus du double le taux d'abandon au bout de 6 mois comparativement au placébo. Il existe actuellement des programmes de médicaments subventionnés dans les Territoires-du-Nord-Ouest, en Ontario, en Alberta, en Saskatchewan, et au Québec.

On compte environ 550000 fumeurs en ColombieBritannique. ${ }^{1}$ Au cours des 12 mois ayant suivi la mise en œuvre du programme de lutte contre le tabagisme, il y a eu presque 150000 demandes de TRN gratuite, et plus de 40000 fumeurs ont eu droit à un remboursement partiel du coût des médicaments de désaccoutumance au tabac sur ordonnance. ${ }^{10}$ Ces chiffres démontrent qu'un grand nombre de personnes veulent abandonner le tabac et qu'elles recherchent de l'aide pour y parvenir.

Tiré de Department of Emergency Medicine, Vancouver General Hospital, and Faculty of Medicine, University of British Columbia, Vancouver, BC.

Correspondance à: Dr. Ka Wai Cheung, Department of Emergency Medicine, Vancouver General Hospital, 855 West 12th Avenue, Vancouver, BC V5Z 1M9; tokwcheung@gmail.com.

Cet article a été évalué par des pairs. 
Dans le cadre d'un essai comparatif à répartition aléatoire (NCT01454375) mené actuellement au SU du Vancouver General Hospital (VGH), il se fait une collecte de données sur l'utilisation de la TRN, du bupropion ou de la varénicline parmi les fumeurs qui viennent consulter. Le VGH est un hôpital d'enseignement, de soins tertiaires, qui répond aux besoins d'une population urbaine, et le SU traite plus de 80000 patients par année. Bien que le programme de lutte contre le tabagisme ait connu un grand succès dans la population générale de fumeurs en ColombieBritannique, il n'en va pas de même pour la population de patients vue au service des urgences. Depuis la mise en œuvre du programme il y a 12 mois, l'utilisation des aides à la désaccoutumance au tabac au sein de la population à l'étude au SU du VGH reste faible; en effet, environ $6 \%$ de cette population a recours à la TRN et moins de $1 \%$ utilise le bupropion ou la varénicline.

La population de patients traitée aux SU représente un groupe fortement prédisposé aux maladies, et elle connaît une prévalence du tabagisme plus élevée que la population générale. ${ }^{11}$ Il se peut que bon nombre de patients qui consultent aux SU aient moins facilement accès aux services de santé, par exemple aux médecins de famille. Ainsi, les SU deviennent de plus en plus le «filet de sécurité» de tout le système de soins de santé, ${ }^{11}$ et les consultations aux SU peuvent être la seule occasion, pour les patients concernés, d'avoir accès aux programmes de santé communautaire. Point important à souligner: les fumeurs qui reçoivent des conseils pendant qu'ils sont malades sont plus disposés à abandonner le tabac que ceux qui reçoivent des conseils lorsqu'ils sont en bonne santé; c'est ce qu'on appelle la «période propice à l'apprentissage».12 Enfin, les fumeurs qui consultent aux SU veulent cesser de fumer; d'après une étude menée dans des SU, environ $70 \%$ des fumeurs désirent renoncer au tabac, et presque $50 \%$ veulent se défaire de leur habitude en l'espace de 1 mois. $^{13}$

Le personnel des SU a donc l'occasion de jouer un rôle crucial dans les interventions de désaccoutumance au tabac et dans l'établissement de liens avec des programmes de santé communautaire très importants. Les interventions de désaccoutumance au tabac sont rentables, même plus que les interventions cliniques courantes comme l'abaissement du taux de cholestérol à lipoprotéines de basse densité et la régulation de la pression artérielle. ${ }^{14}$ Des recherches sont en cours afin d'améliorer les moyens de dépister les fumeurs aux SU et de leur prodiguer des conseils. Malheureusement, non seulement les patients aux SU ne connaissent-ils pas l'existence de ressources communautaires, mais il peut en être de même pour le personnel des SU. Si on pouvait entrer en relation avec la population de patients vue aux SU, l'augmentation du taux de désaccoutumance au tabac à l'aide de la TRN et d'autres médicaments pourrait se traduire par une diminution importante de la morbidité et de la mortalité liées au tabagisme et par des réductions substantielles de coût. Le personnel des SU peut jouer un rôle crucial dans les interventions de désaccoutumance au tabac, et il est pressant d'approfondir la recherche afin que soient améliorés les moyens de sensibilisation du personnel des SU aux programmes communautaires et d'orientation des patients examinés aux SU vers ces ressources.

Concurrents: Aucun déclaré.

Mots clés: services des urgences, tabac, thérapie de remplacement de la nicotine

\section{RÉFÉRENCES}

1. Propel Centre for Population Health Impact. Tobacco use in Canada: patterns and trends - 2012 edition. Available at: www. tobaccoreport.ca/2012 (accessed January 15, 2013).

2. Health Canada. 2011 Canadian Tobacco Use Monitoring Survey. Available at: http://www.hc-sc.gc.ca/hc-ps/tobac-tabac/ research-recherche/stat/ctums-esutc_2011-eng.php (accessed January 10, 2013).

3. Canadian Public Health Association. Canadian Public Health Association position paper. The winnable battle: ending tobacco use in Canada. December 2011. Available at: http://www.cpha. ca/uploads/positions/position-paper-tobacco_e.pdf (accessed January 15, 2013).

4. British Columbia Ministry of Health. B.C. Smoking Cessation Program. 2011. Available at: http://www.health.gov.bc.ca/ pharmacare/stop-smoking/ (accessed April 15).

5. Alpert HR, Connolly GN, Biener L. A prospective cohort study challenging the effectiveness of population-based medical intervention for smoking cessation. Tobacco Control 2013;22:32-7, doi:10.1136/tobaccocontrol-2011-050129.

6. Moore D, Aveyard P, Connock M, et al. Effectiveness and safety of nicotine replacement therapy assisted reduction to stop smoking: systematic review and meta-analysis. BM7 2009;338:867-79, doi:10.1136/bmj.b867.

7. Eisenberg MJ, Filion KB, Yavin D, et al. Pharmacotherapies for smoking cessation: a meta-analysis of randomized controlled trials. CMA7 2008;179:135-44, doi:10.1503/ cmaj.070256.

8. Cahill K, Stead LF, Lancaster T. Nicotine receptor partial agonists for smoking cessation [update appears in Cochrane Database Syst Rev 2010;(12):CD006103]. Cochrane Database Syst Rev 2008;(3):CD006103. 
9. Hughes J, Stead L, Lancaster T. Antidepressants for smoking cessation [update appears in Cochrane Database Syst Rev 2007;(1):CD000031]. Cochrane Database Syst Rev 2004;(4):CD000031.

10. BC Government Online News Source. Newsroom. Celebrating the anniversary of the Smoking Cessation Program. October 12, 2012. Available at: http://www.newsroom.gov. bc.ca/2012/10/celebrating-the-anniversary-of-the-smokingcessation-program.html (accessed January 15, 2013).

11. Cummings GE, Francescutti LH, Predy G, et al. Health promotion and disease prevention in the emergency department: a feasibility study. CFEM 2006;8:100-5.

12. Baker DW, Stevens CD, Brook RH. Regular source of ambulatory care and medical care utilization by patients presenting to a public hospital emergency department.
FAMA 1994;271:1909-12, doi:10.1001/jama.1994.035104 80033030.

13. Bernstein SL, Becker BM. Preventive care in the emergency department: diagnosis and management of smoking and smoking-related illness in the emergency department: a systematic review. Acad Emerg Med 2002;9:720-9, doi:10. 1111/j.1553-2712.2002.tb02153.x.

14. Lowenstein SR, Tomlinson D, Koziol-McLain J, et al. Smoking habits of emergency department patients: an opportunity for disease prevention. Acad Emerg Med 1995; 2:165-71, doi:10.1111/j.1553-2712.1995.tb03189.x.

15. Kahn R, Robertson RM, Smith $\mathrm{R}$, et al. The impact of prevention on reducing the burden of cardiovascular disease. Circulation 2008;118:576-85, doi:10.1161/CIRCULATION AHA.108.190186. 